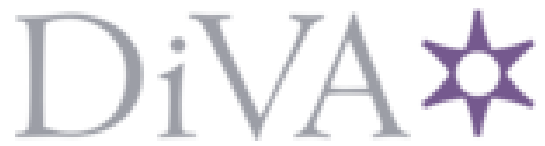

http://www.diva-portal.org

Postprint

This is the accepted version of a paper presented at Konferens.

Citation for the original published paper:

Yao, Y., Popescu, A., Fiedler, M., Ljung, R. (2017)

On the Performance of Video Streaming in Energy-Aware Wireless Mesh Networks.

In:

N.B. When citing this work, cite the original published paper.

Permanent link to this version:

http://urn.kb.se/resolve?urn=urn:nbn:se:bth-15640 


\section{On the Performance of Video Streaming in Energy-Aware Wireless Mesh Networks}

\author{
Yong Yao, Adrian Popescu and Markus Fiedler \\ Blekinge Institute of Technology, Karlskrona, Sweden \\ Email: \{yong.yao, adrian.popescu, markus.fiedler\}@bth.se
}

\author{
Rickard Ljung \\ Sony Mobile Communications, Lund, Sweden \\ Email: rickard.ljung@ sonymobile.com
}

\begin{abstract}
Mobile multimedia has today become a promising application for end users and service providers. With reference to the existing systems for mobile communications, this application further demands for solving several technical problems, especially regarding video streaming over wireless networks. An interesting approach is in form of Wireless Mesh Network (WMN) based networks, where the individual video flows operate in an end-to-end (e2e) manner along a particular networking scenario including several mesh routers. That means, a particular mesh router may be traversed by multiple video flows. This situation may become even more complicated in the case of a large amount of packet retransmissions, which may deteriorate the performance of video flows. To investigate this problem, a two-level Modulated Markov Poisson Process (MMPP) based queueing model is built up and the transport performance of e2e video streaming in WMN based mobile multimedia system is analysed. Four metrics are used to study the system performance, namely e2e throughput, e2e delay, e2e error-rate and traffic-related energy consumption. Numerical analysis and evaluation studies are done. Based on the reported results, two different solutions are suggested and discussed with regard to the trade-off among these metrics.
\end{abstract}

\section{INTRODUCTION}

With the recent explosive growth of mobile devices, various mobile applications are appearing on the market. This situation raises the need of ubiquitous access for end users over wireless networks. An interesting evolution is the use of mesh networks together with infrastructures such as $4 \mathrm{G} / 5 \mathrm{G}$ networks. This started with public safety solutions but evolution beyond such services are expected to come, e.g., for device-to-device communication [1] and also for improving the effective link budget by means of mesh network (e.g., for extended coverage of cellular networks). In a long-term time period, the use of mesh networks may increase in various ICT areas such as home networks. This is because industrial leaders such as Google and Netgear now start introducing multiple Wi-Fi access points as a corresponding default solution.

Mobile multimedia over Wireless Mesh Network (WMN) has become an important application for service providers and end users. Mobile multimedia usually refers to multiple video flows, each of which is defined to be an end-to-end (e2e) data transmission from the video streaming source side (e.g., a gateway router) to the video consumer side (e.g., a mobile terminal). Designing efficient WMN for e2e video streaming is a complicated procedure also with the need for provision of Quality of Service (QoS) for end users.

978-1-5386-3873-6/17/\$31.00 (C) 2017 IEEE
Connected with this, a challenging problem is in the form of demand for reducing the power consumption in WMNs while maintaining the acceptable QoS performance [2], [3]. One needs to model and to measure the QoS and energy consumption for e2e video streaming in WMNs. Along with this research line, much work has been recently reported. For instance, the authors of [5] suggest an energy-aware routing algorithm for WMNs to provide the balance between energy savings and maintaining the quality of video content. In [6], the authors suggest a new relay selection mechanism for efficient video broadcasting in WMNs. This mechanism uses the Multiple Description Coding (MDC) technique with joint consideration of transmission power allocation at relay stations. In [9], the authors suggest a numerical method to model the power consumption of network elements like, e.g., switch, router. This also provides an estimation based approach to measure the energy consumed by the network traffic through the network elements. In [8], the authors suggest an energyaware MAC-layer solution for WMNs targeted to balance the energy-saving and QoS at the devices deployed in WMNS.

These studies lay the ground for studying the energy-aware WMNs for mobile multimedia system. However, most of this work is focused on the design of efficient MAC-layer solution for e2e routing. To the best of our knowledge, there are few studies done so far on investigating the video traffic related energy consumption with focus on configurations of traffic priority and packet buffering at the Mesh Router (MR) side. In this paper, we study the effect of these two factors on the e2e video traffic related energy consumption in WMNs. We build up a two-level MMPP queueing model, based on which numerical performance analysis and energy consumption estimation are conducted.

The paper is reported as a partial result of the research project Consumption OptimizatioN in VIdeo NEtworks (CONVInCE) [4]. This is a three years Celtic-Plus project focused on the study and analysis of optimized solution for energy saving in IP-based video distribution networks.

The rest of paper is as follows. Section II describes the system model. Estimation of video traffic related e2e energy consumption is presented in Section III. A two-level MMPP queueing model is built up in Section IV. The numerical based performance evaluation is conducted in Section V. Finally, we conclude the paper in Section VI. 


\section{SYSTEM MODEL}

We consider a multimedia WMN consisting of a single Gateway Router (GR) like, e.g., a WiFi access point, and a set of Mesh Routers (MRs). The GR is typically connected to Internet, and it transmits the video data in form of packet to its neighbouring MRs. Furthermore, there is a set of available channels used for wireless transmission among the GR and MRs.

\section{A. Video Streaming Model}

The MR is responsible for two operations: i) relaying the video packets from/to its neighbouring MRs, and ii) transmitting the video packets to destination mobile devices located within its radio range. These two operations are assumed to be done in two different channels allocated from the abovementioned set of channels. The channel used for the first operation is called Allocated Channel for Relay (AC-R). The channel used for doing the second operation is called Allocated Channel for Local (AC-L). For all MRs in the modelled system, channel allocation is assumed to be done by using protocols for, e.g., information exchange among MRs.

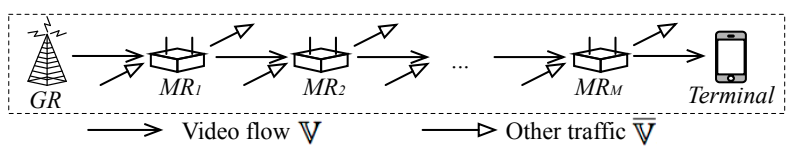

Fig. 1. Uplink transmission along a particular e2e routing path.

As shown in Figure 1, we consider a particular video flow $\mathbb{V}$, which streams along with an e2e routing path $\mathbb{P}$. The routing path consists of $M$ MRs labeled by $R_{1}, R_{2}, \ldots, R_{m}, \ldots, R_{M}$. Let $\mathbb{R}$ and $\mathbb{C}$ denote the sets of $M$ MRs and their channels, respectively. For a particular MR $R_{m} \in \mathbb{R}$, its AC-R and AC-L channels are denoted by $C_{m, R}$ and $C_{m, L}$, respectively, where $C_{m, R}, C_{m, L} \in \mathbb{C}$.

For a particular destination mobile device within the radio range of MR $R_{m}$, the e2e video streaming is defined to be the procedure of transmitting packets from the GR to this particular mobile device. The packet transmission through the related MRs is assumed to be constrained as follows:

- MR $R_{m}$ uses channel $C_{m, R}$ to transmit video packets to the MR $R_{m+1}$ for $0 \leq m<M$, or to the destination mobile device for $m=M$.

- If $0 \leq m<m^{\prime} \leq M$, each MR $R_{m^{\prime}}$ provides relay service to the video packet until it reaches the destination mobile device.

\section{B. Transmission Model of Mesh Router}

In the considered WMNS, the MR $R_{m}$ needs to concurrently deal with different traffics of video flows, which are targeted at different destination mobile devices. This directly influences the transmission performance of the video flow $\mathbb{V}$ along the routing path $\mathbb{P}$. To investigate this effect, we first classify the traffics arrived at MR $R_{m}$ into two categories. The first category of traffic is associated with the video flow. While, the second category is related to other traffic flows, which may also include other video flows. These two categories of traffic are denoted by $\mathbb{V}$ and $\overline{\mathbb{V}}$, respectively.

At the MR $R_{m}$, the packet arrivals of video flow $\mathbb{V}$ are assumed to follow the Poisson process with average rate $\lambda_{m}^{(0)}$. Similarly, the packets arrivals associated to traffic flow $\overline{\mathbb{V}}$ are assumed to follow the Poisson process with average rate $\lambda_{m}^{(1)}$. Subsequently, the packet arrivals of all traffic flows at MR $R_{m}$ can be assumed to follow a two-state Markov-modulated Poisson Process $\left(\mathrm{MMPP}_{2}\right)$. In our study, $\mathrm{MMPP}_{2}$ provides a feasible approach to model the traffic flows through MR $R_{m}$ as a modulated process with average rate alternating between two values $\lambda_{m}^{(0)}$ and $\lambda_{m}^{(1)}$. These two values are also referred to as two states of the $\mathrm{MMPP}_{2}$ model. Further, we let $\mathbb{Q}=$ $\left\{q_{01}, q_{10}\right\}$ denote the generator matrix for the state transitions between these two values.

The MR $R_{m}$ is also responsible for relaying the arrived packets to its neighbouring MRs. Further, the relaying process is assumed to operate on an exponential distribution with average rate $\mu_{m}$ for any arbitrary packet.

\section{Video TRAFFiC RElated ENERGy CONSUMPTION}

In this section, the energy consumption of video flow $\mathbb{V}$ along with the routing path $\mathbb{P}$ is analysed. For simplicity purposes, we consider a particular time interval $[t, t+\Delta t]$ to be partitioned into $N$ identical unit time slots. Each time slot has the length $\delta$, where $\Delta t=N \delta$. Further, let $t_{n}$ denote a particular time moment $(t+n \delta)$, where $n \in\{1,2, \ldots, N\}$.

\section{A. Power Consumption Model}

During the time slot $\left(t_{n}, t_{n+1}\right]$, the power consumption (expressed in Watts or Joules/sec) measured on MR $R_{m} \in \mathbb{R}$ consists of three parts of power consumptions.

The first part is the power used for MR $R_{m}$ under the situation of no traffic flow through it, i.e., the so-called idle power state. The second and the third parts are the power used for transmitting the packets during the given unit time interval (e.g., per second) for two video flows $\mathbb{V}$ and $\overline{\mathbb{V}}$, respectively. The later two parts of power consumption are also called video flow related powers, and they are denoted by $P_{m, n}$ and $\bar{P}_{m, n}$.

Usually, information about the idle power of MRs can be obtained from testing experiments, or the corresponding manufactures provide it. Hence, the focus of e2e power consumption is laid on the video flow related power consumption of MRs. In the following we first derive the calculation of $P_{m, n}$. The calculation of $\bar{P}_{m, n}$ can then be derived in the same way.

From the packet transmission point of view, $P_{m, n}$ is related to two different operations on the video flow $\mathbb{V}$. They are: i) storing and forwarding data (i.e., in terms of multiple packets), and ii) processing packets. The corresponding power consumptions are denoted by $P_{m, n}^{\dagger}$ and $P_{m, n}^{\ddagger}$. That means $P_{m, n}=P_{m, n}^{\dagger}+P_{m, n}^{\ddagger}$.

\section{B. Energy Consumption Model}

The calculation of energy consumption used by a given MR $R_{m}$ to deal with video flows is as follows. The 
above-mentioned two operations have different responsibilities, which lead to different ways for quantifying the energy consumptions. The energy used for storing and forwarding packets is associated to the size of data transmitted in a time slot. The energy used for processing packets is associated with the number of packets transmitted in a time slot.

During the time slot $\left[t_{n}, t_{n+1}\right]$, let $E_{m, n}^{\dagger}$ denote the energy consumption used by device $R_{m}$ to store and to forward video flow $\mathbb{V}$. Further, let $E_{m, n}^{\ddagger}$ denote the energy consumption for processing packets. With reference to the equation $P(W)=$ $E(J / b i t) R(b i t / s)$, we have

$$
\begin{aligned}
P_{m, n}^{\dagger} & =E_{m, n}^{\dagger} \gamma_{m, n} \\
P_{m, n}^{\ddagger} & =\left[E_{m, n}^{\ddagger} \gamma_{m, n}\right] / l_{\mathbb{V}}
\end{aligned}
$$

where $\gamma_{m, n}$ is defined to be the data transmission throughput at device $R_{m}$ during the time slot $\left[t_{k}, t_{k+1}\right]$. Further, $l_{\mathbb{V}}$ denotes the uniform size of each packet belonging to the video flow $\mathbb{V}$. Connected to the metric $\gamma_{m, n}$, we introduce another metric $\beta_{m, n}$, which is called packet rate (i.e., packets/s) and satisfies $\beta_{m, n}=\gamma_{m, n} / l_{\mathbb{V}}$.

Two equations (1) and (2) further create a new metric for measuring the energy used by MR $R_{m}$ to deal with the video flow $\mathbb{V}$. This new metric is called incremental energy per packet. It is denoted by $E_{m, n}^{(p k t)}$ and it is computed by:

$$
E_{m, n}^{(p k t)}=l_{\mathbb{V}} E_{m, n}^{\dagger}+E_{m, n}^{\ddagger}
$$

Subsequently, we can compute the video flow related power consumption at the MR $R_{m}$ by:

$$
P_{m, n}=E_{m, n}^{(p k t)} \beta_{m, n}
$$

For the time interval $[t, t+\Delta t]$, where $\Delta t=N \delta$, let $\tilde{E}_{m, \Delta t}$ denote the energy consumed by MR $R_{m}$ to deal with the video flow $\mathbb{V}$. $\tilde{E}_{m, \Delta t}$ is computed by:

$$
\tilde{E}_{m, \Delta t}=\sum_{n=1}^{N}\left[\delta P_{m, n}\right]=\sum_{n=1}^{N}\left[\delta E_{m, n}^{(p k t)} \beta_{m, n}\right]
$$

Furthermore, we assume that the value of $E_{m, n}^{(p k t)}$ at MR $R_{m}$ does not change during the time interval $[t, t+\Delta t]$. This is an assumption used in e2e networking system [9], where the value of $E_{m, n}^{(p k t)}$ is practically measured as a nearly constant value. $\tilde{E}_{m, \Delta t}$ is therefore given by:

$$
\tilde{E}_{m, \Delta t} \stackrel{\delta \rightarrow 0}{=} \bar{E}_{m, \Delta t}^{(p k t)} \int_{\tau=t}^{t+\Delta t} \beta_{m, \tau} d \tau=\bar{E}_{m, \Delta t}^{(p k t)} \mathcal{T}_{m}
$$

where $\mathcal{T}_{m}$ denotes the average rate of packets of video flow $\mathbb{V}$ successfully transmitted from MR $R_{m}$ to MR $R_{m+1}$. It also indicates the throughput of video flow $\mathbb{V}$ from $R_{m}$ to $R_{m+1}$.

We further assume that all MRs have identical characterization regarding incremental energy per packet. In other words, we let $\bar{E}_{m, \Delta t}^{(p k t)}$ equal to $\bar{E}_{\Delta t}^{(p k t)}$. The energy used by all $M$ MRs along the routing path $\mathbb{P}$ to deal with the video flow $\mathbb{V}$ is therefore:

$$
\tilde{E}_{\Delta t}=\sum_{m=1}^{M} \tilde{E}_{m, \Delta t}=\bar{E}_{\Delta t}^{(p k t)} \sum_{m=1}^{M} \mathcal{T}_{m}
$$

\section{Queueing Modeling And Analysis}

Given the above described models, the Markov chains based queueing analysis, together with the formulation of corresponding performance metrics is as follows.

\section{A. Single Mesh Router}

Packet transmission of each MR in the set $\mathbb{R}$ is independent from each other. For a particular MR $R_{k}$, we build up a $\mathrm{MMPP}_{2} / \mathrm{M} / 1$ model with finite buffer length $h_{m}$.

Let the set of three integers $(i, j, k)$ denote the system state of MR $R_{m}$ for dealing with video packets. Specifically, $i$ means the number of packets served by MR $R_{k}$ for the video flows $\mathbb{V}$ and $\overline{\mathbb{V}}$, respectively. While, $j$ indicates one of two particular states of the $\mathrm{MMPP}_{2}$ model described above.

We also let $\pi_{i, j, k}^{(m)}$ denote the steady-state probability of MR $R_{m}$ being in state $(i, j, k)$. We define the system state space as $S_{m}=\{(i, j, k)\}$, where $i, j$ and $k$ are constrained by $i \in$ $\left[0, h_{m}+1\right], j \in\left[0, h_{m}+1\right],(i+j) \in\left[0, h_{m}+1\right]$ and $k=\{0,1\}$.

If $(i, j, k) \in S_{m}, \pi_{i, j, k}^{(m)}$ is in the value range $(0.0,1.0)$. Otherwise, $\pi_{i, j, k}^{(m)}$ is equal to zero. Further, the rate of transition flow into a state $(i, j, k)$ must be equal to the rate of transition flow out of this state. We illustrate the state transition diagram in Fig. 2.

As observed in this figure, the state transition of the system is triggered by two activities. They are the arrival of new video packet belonging to video flow $\mathbb{V}$ and the arrival of new video packet belonging to other video traffics as well as the transition change between these two activities. The corresponding balance equations are:

$$
\begin{aligned}
& \pi_{i, j, 0}^{(m)}\left(\lambda_{m}^{(0)} \theta_{i, j, 0}+\mu_{m} \phi_{i, j, 0}+q_{01}\right) \\
= & \pi_{i-1, j, 0}^{(m)} \lambda_{m}^{(0)}+\pi_{i+1, j, 0}^{(m)} \mu_{k}+\pi_{i, j, 1}^{(m)} q_{10}, \text { and } \\
& \pi_{i, j, 1}^{(m)}\left(\lambda_{m}^{(0)} \theta_{i, j, 1}+\mu_{m} \phi_{i, j, 1}+q_{10}\right) \\
= & \pi_{i, j-1,0}^{(m)} \lambda_{m}^{(1)}+\pi_{i, j+1,0}^{(m)} \mu_{k}+\pi_{i, j, 0}^{(m)} q_{01}
\end{aligned}
$$

where $\pi_{i, j, k}^{(m)}$ is equal to zero if $(i, j, k) \notin \mathbb{S}_{m}$. The values of $\theta_{i, j, 0}, \phi_{i, j, 0}, \theta_{i, j, 1}, \phi_{i, j, 1}$ are constrained as follows:

$$
\begin{aligned}
& \theta_{i, j, 0}, \phi_{i, j, 0}= \begin{cases}1, & 0 \leq i+j<h_{m}+1 \\
0, & \text { otherwise }\end{cases} \\
& \theta_{i, j, 1}, \phi_{i, j, 1}= \begin{cases}1, & 0<i+j \leq h_{m}+1 \\
0, & \text { otherwise }\end{cases}
\end{aligned}
$$

Further, we sum up all steady-state probabilities in conjunction with the equation $\sum_{\forall i, j, k}^{(i, j, k) \in S}\left[\pi_{i, j, k}^{(m)}\right]=1$. By combining the above equations $(8-9)$, we can construct a set of linear equations. If the values of $\lambda_{m}^{(0)}$ and $\lambda_{m}^{(1)}$ are already known, we can compute the steady-state probabilities of all states for a particular mesh router MR $R_{m} \in \mathbb{R}$.

\section{B. Mesh Routing}

For the video flow $\mathbb{V}$ along with the routing path $\mathbb{P}$, the packet arrival rate at the $R_{m} \in \mathbb{R}$, i.e., $\lambda_{m}^{(0)}$, depends on the transmission rate of packets from the MR $R_{m-1}$ to the MR $R_{m}$. Every MR in the set $\mathbb{R}$ has finite buffer, thus leading 


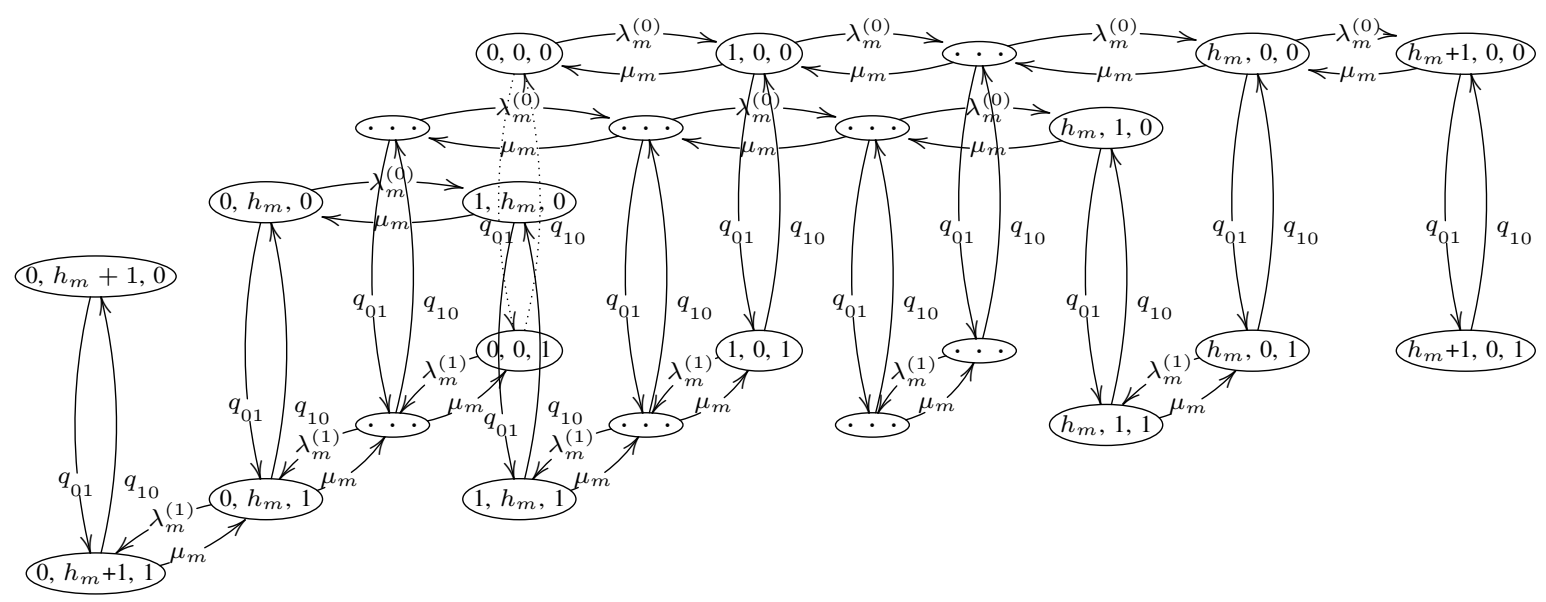

Fig. 2. State diagram of MR $R_{m}$ at steady-state.

to the event that some arrived video packets are blocked. For particular MR $R_{m}$, the event of blocking the packets of video flow $\mathbb{V}$ occurs for system state satisfying $i=h_{m}$ and $j=0$. Let $\mathcal{P}_{b l, m}^{(0)}$ denote the blocking probability. It is computed by:

$$
\mathcal{P}_{b l, m}^{(0)}=\lambda_{m}^{(0)} \sum_{\forall i, j, k}^{(i, j, k) \in \mathbb{S}_{m}}\left[\pi_{i, j, k}^{(m)} \mid i+j=h_{m+1}\right]
$$

The above addressed blocking event indicates that the packet arrival rate at a particular MR actually depends on its neighbouring relay MRs. For the video flow $\mathbb{V}$ along with the routing path $\mathbb{P}$, we therefore compute the arrival rate of the packets from MR $R_{m-1}$ to MR $R_{m}$ as $\lambda_{m}^{(0)}=\lambda_{m-1}^{(0)}\left(1-\mathcal{P}_{b l, m-1}^{(0)}\right)$.

As shown in Fig. 2, the packet arrival rate of video flow $\mathbb{V}$ at the MR $R_{m}$ is computed by:

$$
\lambda_{m}^{(0)}=\lambda_{1}^{(0)} \prod_{m=1}^{m-1}\left(1-\mathcal{P}_{b l, m}^{(0)}\right)
$$

For all MRs $R_{m} \in \mathbb{R}$, we can formalize the steady-state vector for all MR as $\left\{\boldsymbol{\Pi}_{1}, \boldsymbol{\Pi}_{2}, \ldots, \boldsymbol{\Pi}_{m}, \ldots, \boldsymbol{\Pi}_{M}\right\}$, where $\boldsymbol{\Pi}_{m}=$ $\left\{\pi_{i, j, k}^{(m)} \mid(i, j, k) \in \mathbb{S}_{m}\right\}$. By substituting the equation (13) into equations $(8-9)$, the steady-state probability in the steadystate vector can be sequentially computed from $\Pi_{1}$ to $\Pi_{M}$.

\section{Performance Metrics}

Three performance metrics are studied as follows.

1) E2e throughput: for a given video stream $\mathbb{V}$, the e2e throughput is defined to be the average rate of packets successfully transmitted from GR to the destination mobile device. Let $\mathcal{T}$ denote the e2e throughout, which is computed by:

$$
\mathcal{T}=\lambda_{1}^{(0)} \prod_{m=1}^{M}\left(1-\mathcal{P}_{b l, m}^{(0)}\right)
$$

2) Average e2e delay: for a given packet, this metric represents the total time spent by packet for the transmission from the GR to the destination mobile device. This is the sum of the waiting time of this particular packet at each MR. For the video stream $\mathbb{V}$, let $\mathcal{D}_{m}$ denote the average waiting time experienced by an arbitrary packet at MR $R_{m} \in \mathbb{R}$, which includes the time for queueing and for transmission. To compute $\mathcal{D}_{m}$, we need to consider the average number of packets belonging to video stream $\mathbb{V}$ in $\mathrm{MR} R_{m}$, which is given by $\mathcal{N}_{m}=\sum_{\forall i, j, k}^{(i, j, k) \in \mathbb{S}_{m}}\left[(i+j)\left(\pi_{i, j, 0}^{(m)}+\pi_{i, j, 1}^{(m)}\right)\right]$. According to the Little's Theorem, we obtain $\mathcal{D}_{m}=\mathcal{N}_{m} / \lambda_{m}^{(0)}$. Subsequently, the average e2e transmission delay is given by:

$$
\overline{\mathcal{D}}=\sum_{m=1}^{M} \mathcal{D}_{m}=\sum_{m=1}^{M}\left[\frac{\mathcal{N}_{m}}{\lambda_{m}^{(0)}}\right]
$$

3) E2e error rate: for the video stream $\mathbb{V}$, the individual packet needs to be transmitted from GR to the destination mobile device. The transmission error occurs when a particular packet is blocked at a MR $R_{m} \in \mathbb{R}$. Such blocking event may happen due to the limited buffer slots for queueing packets.

For video stream $\mathbb{V}$, we let $\alpha_{M}$ denote the percentage of packets blocked by different MRs along the path $\mathbb{P}$. By considering the packet arrival rate $\lambda_{1}^{(0)}$ from the GR to the first MR $R_{1}$, the relationship between $\lambda_{1}^{(0)}$ and the above-computed e2e throughput can be represented as $\mathcal{T}=\left(1-\alpha_{M}\right) \lambda_{1}^{(0)}$. Hence, $\alpha_{M}$ is computed by:

$$
\alpha_{M}=1-\frac{\mathcal{T}}{\lambda_{1}^{(0)}}=1-\prod_{m=1}^{M}\left(1-\mathcal{P}_{b l, m}^{(0)}\right)
$$

4) Average end-to-end energy consumption: for the video stream $\mathbb{V}$, this metric is defined to be the total energy used by all $M$ MRs to transmit video packets from GR to destination mobile during an unit time interval. This metric is namely equivalent to the denotation $\tilde{E}_{\Delta t}$ used in equation (7).

Given that the packets arrived at MR $R_{m}$ may be blocked for further transmission, the variable $\mathcal{T}_{m}$ in equation (7) is equal to $\lambda_{m}^{(0)}\left(1-\mathcal{P}_{b l, m}^{(0)}\right)$. Subsequently, the equation is reformulated as:

$$
\theta_{M}=\frac{\tilde{E}_{\Delta t}}{\bar{E}_{\Delta t}^{(p k t)}}=\sum_{m=1}^{M}\left[\lambda_{m}^{(0)}\left(1-\mathcal{P}_{b l, m}^{(0)}\right)\right]
$$

where the new metric $\theta_{M}$ is called e2e traffic-energy coefficient. Equation (17) also indicates the need of providing the 
tradeoff between e2e throughput and e2e energy consumption. Specifically, the e2e throughput may be constrained by the so-called sustainable throughput due to different constraints (e.g., limited bandwidth) along the routing path [11]. For this particular case, the e2e energy consumption can be reduced by accordingly jointly altering related parameters such as arrival rate of video packets and the blocking probability of video packets at individual MR in WMNs. The corresponding evaluation scenarios are studied in the following section.

\section{Performance Evaluation}

In the following, performance evaluation results are reported for a system composed of four mesh routers, i.e., $\mathbb{R}=\left\{R_{m}\right\}$, where $m \in\{1,2,3,4\}$. The evaluation results and associated discussion are as follows.

\section{A. Evaluation Scenarios}

Three different evaluation scenarios are designed and conducted for specific research goals. For all three scenarios, the number of queueing slots at each MR, i.e., $h_{m}$, is configured to equal the same value $h$. Further, the packet service rate at each MR is configured to be a constant value, i.e., $\mu_{m}=1.0$. Other parameter settings are reported in Table I. The goals of three evaluation scenarios are as follows:

- $1^{\text {st }}$ scenario: study of the effect of queueing slots $h_{m}$ on the system performance.

- $2^{\text {nd }}$ scenario: study of the effect of transmission ratio between $q_{10}$ and $q_{01}$ on the system performance.

- $3^{\text {rd }}$ scenario: study of the effect of packet arrival rate of other traffic flows, i.e., $\lambda_{m}^{(1)}$, on the system performance.

TABLE I

PARAMETER SETTINGS

\begin{tabular}{|c|c|c|}
\hline Scenario & \multicolumn{2}{|c|}{ Parameters } \\
\hline \multirow{2}{*}{$1^{\text {st }}$} & Special & $h_{m}=h, h \in\{2,4,6,8,10\}$ \\
\cline { 2 - 3 } & Others & $\lambda_{1}^{(0)}=0.3, \lambda_{m}^{(1)}=0.4, q_{01}=q_{10}=0.5$ \\
\hline \multirow{2}{*}{$2^{\text {nd }}$} & \multirow{2}{*}{ Special } & $q_{10}+q_{01}=1.0$ \\
& & $\left(q_{10}: q_{01}\right) \in\{1: 9,3: 7,5: 5,7: 3,9: 1\}$ \\
\cline { 2 - 3 } & Others & $\lambda_{1}^{(0)}=0.3, \lambda_{m}^{(1)}=0.4, h=6$ \\
\hline \multirow{2}{*}{$3^{\text {rd }}$} & Special & $\lambda_{m}^{(1)} \in\{0.4,0.5,0.6,0.7,0.8\}$ \\
\cline { 2 - 3 } & Others & $\lambda_{1}^{(0)}=0.3, h=6, q_{10}=q_{01}=0.5$ \\
\hline
\end{tabular}

\section{B. Results and Discussions}

The numerical results are reported in Figs. 3 - 5 for the above mentioned four scenarios, respectively.

For the first scenario, Fig. 3 shows that both e2e throughput $\mathcal{T}$ and e2e delay $\overline{\mathcal{D}}$ increase with the number of queueing slots $h_{m}$ at each MR. The e2e error-rate $\alpha_{M}$ decreases with $h_{m}$. This is because when $h_{m}$ becomes larger, more packets can be queued by every MR. A smaller number of packets are blocked at every MR. Therefore, a larger number of packets can be successfully transmitted from the GR to the terminal. Subsequently, $\mathcal{T}$ is increased and $\alpha_{M}$ is decreased. The cost is the increase of $\overline{\mathcal{D}}$ due to larger average queueing time at each MR.
For the second scenario, Fig. 4 shows that $\mathcal{T}$ increases with the ratio between two state transitions of the $M M P P_{2}$ process, i.e., $q_{01} / q_{10}$. Further, both $\overline{\mathcal{D}}$ and $\alpha_{M}$ decreases with $q_{10} / q_{01}$. This is because larger $q_{10}$ is compared to $q_{01}$, higher priority the video flow $\mathbb{V}$ is given over the traffic $\overline{\mathbb{V}}$ along the routing path $\mathbb{P}$. In other words, there is a higher probability of blocking packets of other traffics $\bar{V}$ at each MR, and thus more packets of video flow $\mathbb{V}$ are accepted. As a result, $\mathcal{T}$ is increased and $\alpha_{M}$ is decreased. Further, when $q_{10} / q_{01}$ becomes larger, more resources of the e2e system are used to deal with video flow $\mathbb{V}$. This situation leads to less e2e delay $\overline{\mathcal{D}}$ for the video flow $\mathbb{V}$

For the third scenarios, Fig. 5 shows that $\mathcal{T}$ decreases with the packet arrival rate of $\mathbb{V}$ at each MR, i.e., $\lambda_{m}^{(1)}$. While, both $\overline{\mathcal{D}}$ and $\alpha_{M}$ increases with $\lambda_{m}^{(1)}$. The reason for this is that there are more packets of $\overline{\mathbb{V}}$ competing for resources at each MR, thus reducing the probability of accepting the packets of $\mathbb{V}$. Therefore, $\mathcal{T}$ is decreased, and both $\overline{\mathcal{D}}$ and $\alpha_{M}$ are increased.

Regarding the energy consumption of video flow $\mathbb{V}$, Figs. 3,4 and 5 indicate that the e2e traffic-energy coefficient $\theta_{M}$ increases with $\mathcal{T}$. This is an expected result in accordance with the equation (17).

The reported results open for a multiple-objective optimization problem for the e2e video streaming in energy-aware WMN. The goal is to provide the best possible trade-off among different performance metrics like, e2e throughput, e2e delay, e2e error-rate and energy consumption. The first and the second evaluation scenarios indicate two solution approaches for solving the optimization problem. In the first scenario, we need to modify the number of queueing slots at each MR. In second scenario, we need to alter the priority of packet acceptance for different traffic flows at each MR. Both solutions can be practically implemented by using different advanced technologies like, e.g., Software Defined Networking (SDN). SDN is defined as an architecture where the control and data planes are decoupled, and the underlying network infrastructure is abstracted from the applications [10].

\section{CONCLUSION}

The paper reports on the end-to-end (e2e) video streaming in Wireless Mesh Networks (WMNs) based system. To investigate the system performance, a two-level Modulated Markov Poisson Process (MMPP) based queueing model is built up. Four transport performance metrics are derived: e2e throughput, e2e delay, e2e error-rate and traffic-related energy consumption. A theoretical approach is advanced to estimate the video traffic related energy consumption along the e2e routing path. Based on the evaluation results, the problem of balancing the transport performance of e2e video streaming and the e2e energy consumption is addressed. Connected to this, two different solutions are suggested for the mesh router in WMNs. They are referred to as the queueing buffering and the packet priority based schemes. Numerical performance evaluation studies are conducted and the evaluation results show the effectiveness of these two schemes. The future work is to extend the suggested solution for relevant networking 


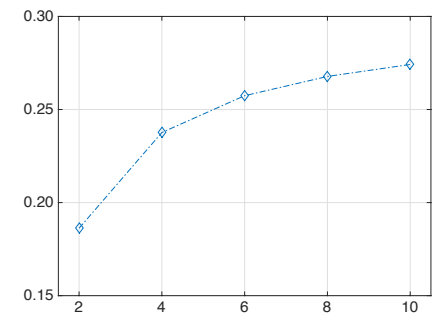

(a) $\mathcal{T}$ v.s. $h$

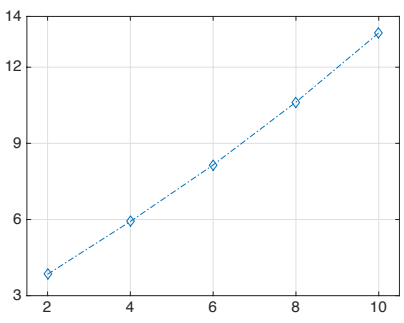

(b) $\overline{\mathcal{D}}$ v.s. $h$

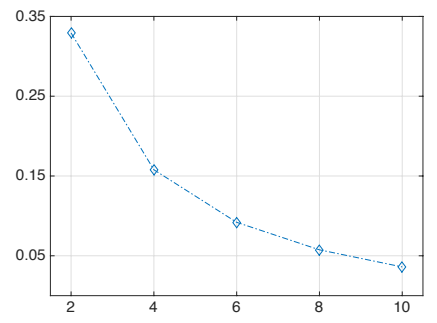

(c) $\alpha_{M}$ v.s. $h$

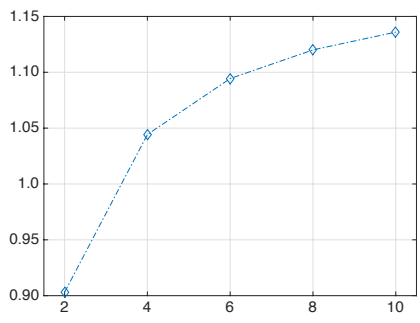

(d) $\theta_{M}$ v.s. $h$

Fig. 3. Numerical results of e2e throughput $\mathcal{T}$, e2e delay $\overline{\mathcal{D}}$, e2e error-rate $\alpha_{M}$, and e2e traffic-energy coefficient $\theta_{M}$ versus queuing slots $h$.

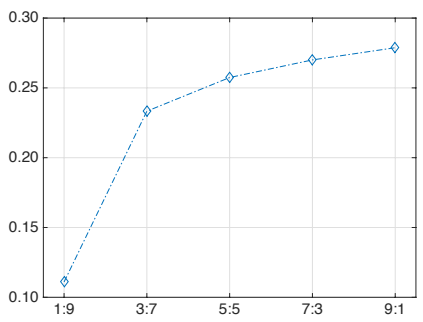

(a) $\mathcal{T}$ v.s. $\left(q_{10} / q_{01}\right)$

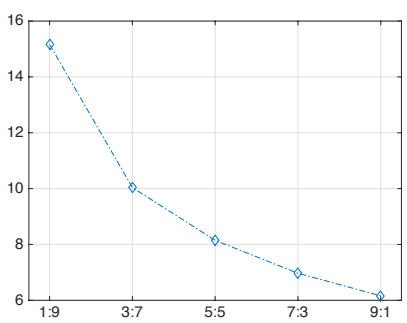

(b) $\overline{\mathcal{D}}$ v.s. $\left(q_{10} / q_{01}\right)$

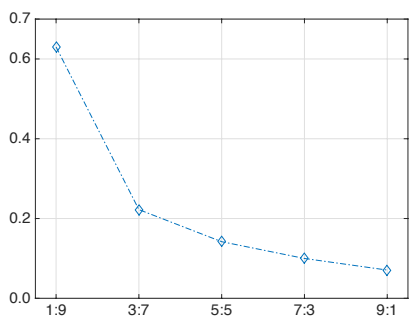

(c) $\alpha_{M}$ v.s. $\left(q_{10} / q_{01}\right)$

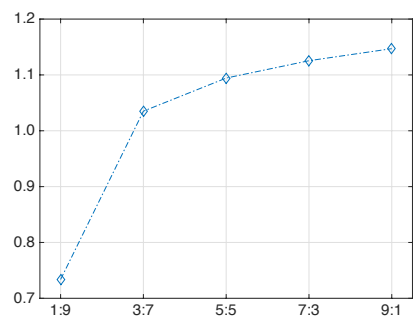

(d) $\theta_{M}$ v.s. $\left(q_{10} / q_{01}\right)$

Fig. 4. Numerical results of e2e throughput $\mathcal{T}$, e2e delay $\overline{\mathcal{D}}$, e2e error-rate $\alpha_{M}$, and e2e traffic-energy coefficient $\theta_{M}$ versus transition ratio $\left(q_{10} / q_{01}\right)$.

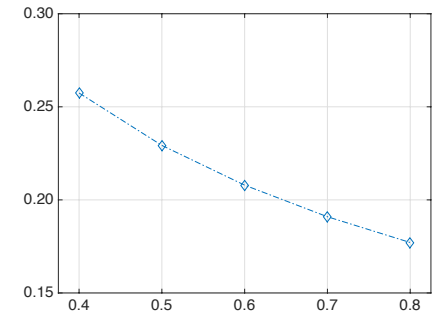

(a) $\mathcal{T}$ v.s. $\lambda^{(0)}$

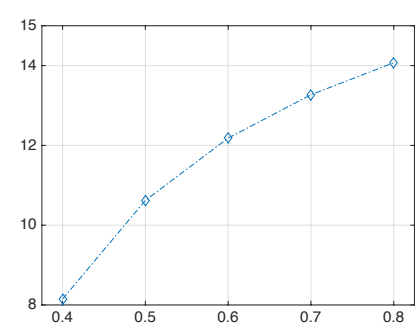

(b) $\overline{\mathcal{D}}$ v.s. $\lambda^{(0)}$

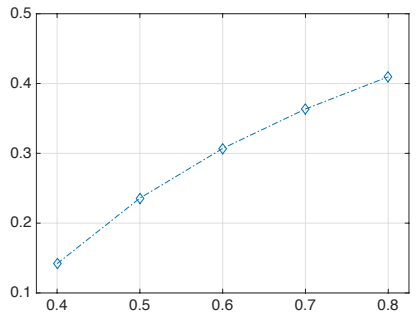

(c) $\alpha_{M}$ v.s. $\lambda^{(0)}$

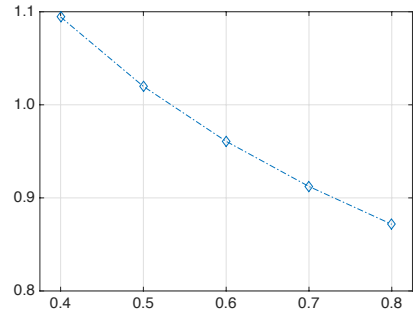

(d) $\theta_{M}$ v.s. $\lambda^{(0)}$

Fig. 5. Numerical results of e2e throughput $\mathcal{T}$, e2e delay $\overline{\mathcal{D}}$, e2e error-rate $\alpha_{M}$, and e2e traffic-energy coefficient $\theta_{M}$ versus video flow packet rate $\lambda^{(0)}$.

scenarios, also including practical video distribution systems like Netflix.

\section{ACKNOWLEDGMENT}

This work is supported by the European Celtic-Plus project CONVINcE and was partially funded by Finland, France, Sweden and Turkey.

\section{REFERENCES}

[1] A. Al-Hourani, S. Kandeepan, E. Hossain, "Relay-Assisted Deviceto-Device Communication: A Stochastic Analysis of Energy Saving", IEEE Transactions on Mobile Computing, Vol. 15, No. 12, December 2016.

[2] European Commission, Horizon 2020 Energy Efficiency, http://ec.europa.eu/easme/en/horizon-2020-energy-efficiency, December, 2015.

[3] A. Popescu, "Greening of IP-Based Video Distribution Networks: Developments and Challenges", IEEE 10th International Conference on Communications, Bucharest, May 2014.

[4] CONVINcE: Consumption Optimization in Video Networks, CELTICPLUS project, http://convince.wp.tem-tsp.eu/celtic-plus/.

[5] S. Chen, Z. Yuan, and G.-M. Muntean, "An Energy-Aware Routing Algorithm for Quality-Oriented Wireless Video Delivery", IEEE Transactions on Broadcasting, vol. 62. no. 1, March 2016.
[6] A. Alganas and D. Zhao, "Relay Station Selection and Power Allocations for Multiple Description-Coded Video in Wireless Mesh Networks", IEEE Wireless Communication and Networking Conference (WCNC), Shanghai, China, April 2013.

[7] A. Hava, Y. G.-D., G.-M. Muntean, and J. Murphy, "Increasing User Perceived Quality by Selective Load Balancing of Video Traffic in Wireless Networks", IEEE Transactions on Broadcasting, vol. 61. no. 2, June 2015.

[8] S. Chen, Z. Yuan, and G.-M. Muntean, "Balancing Energy and Qualityawareness: A MAC-Layer Duty-Cycle Management Solution for Multimedia Delivery over Wireless Mesh Networks", IEEE Transactions on Vehicular Technology, DOI 10.1109, 2016.

[9] Center for Energy Efficient Telecommunications, University of Melbourne, "Measurement based network element power modeling", TREND/GreenTouch Joint Workshop on Green and Energy Efficient Networking, Turin, 2013.

[10] Open Network Foundation, "Software-Defined Networking: The New Norm for Networks", ONF White Paper, April, 2012.

[11] M. Fiedler, A.P. Popescu and Y. Yao, "QoE-Aware Sustainable Throughput for Energy-Efficient Video Streaming”, IEEE International Conference on Sustainable Computing and Communications, Atlanta, USA, October, 2016. 